Article

\title{
Optimization Studies of Photocatalytic Tungsten-Doped Titania Coatings Deposited by Reactive Magnetron Co-Sputtering
}

\section{Marina Ratova *, Glen T. West and Peter J. Kelly}

Surface Engineering Group, School of Engineering, Manchester Metropolitan University, Manchester, M1 5GD, UK; E-Mails: g.west@mmu.ac.uk (G.T.W.); peter.kelly@mmu.ac.uk (P.J.K.)

* Author to whom correspondence should be addressed; E-Mail: marina_ratova@ hotmail.com; Tel.: +44-161-247-4643; Fax: +44-161-247-4693.

Received: 24 August 2013; in revised form: 16 October 2013 / Accepted: 21 October 2013 /

Published: 25 October 2013

\begin{abstract}
In this article we investigate the structural and photocatalytic properties of $\mathrm{W}$-doped titanium dioxide coatings. $\mathrm{TiO}_{2}-\mathrm{W}$ thin films were deposited onto glass slides by reactive magnetron co-sputtering. The properties of the films were analyzed using such techniques as XRD, Raman spectroscopy, EDX, TEM, and surface profilometry. The photocatalytic properties of the coatings were assessed using the methylene blue (MB) degradation test under UV and fluorescent light sources. The methylene blue decomposition experiments showed that, at the optimum dopant concentration of tungsten, the photocatalytic activity can be improved by a factor of 6 , compared to undoped titania. For the coatings discussed within this work, the optimum dopant concentration was determined to be 5.89 at.\% of $\mathrm{W}$. The results indicated that tungsten doping at this level extends the lifetime of the photogenerated charge carriers and significantly increases the photocatalyst surface area.
\end{abstract}

Keywords: titanium dioxide; photocatalytic coatings; doping; methylene blue; magnetron sputtering

\section{Introduction}

Titanium dioxide (or titania) coatings are widely used in photocatalysis due to their low toxicity, high stability, and availability. They find applications in various areas, such as self-cleaning and anti-microbial surfaces, air and water purification facilities, etc. However there are several drawbacks 
associated with the use of titania as a photocatalyst, therefore manufacturers often have to look for alternative materials. The low quantum efficiency of titania is one of the major problems limiting its practical use [1]. In addition, titania has a relatively high band gap value of $3.2 \mathrm{eV}$, which means that it can be activated only with UV light. However for many applications it would be desirable to extend the band gap excitations towards the visible region, and also to prolong the lifetime of photogenerated charge carriers. Doping of titanium dioxide with transition metal ions provides a relatively well-studied and convenient way of solving both problems described above. Titanium dioxide doped with transition metal ions can demonstrate extended band gaps and significantly higher photocatalytic efficiencies [2,3]. The dopant concentration is an important parameter to be considered, as the amount of dopant influences the processes of charge carrier trapping, separation, and recombination [4]. Therefore, the amount of transition metal introduced should be within a so-called optimum concentration, as too low a dopant content does not affect the process of charge carrier generation, and too high a content of doping metal results in the formation of extra recombination sites and shortens the lifetime of photogenerated electrons and holes. Consequently, defining the optimum concentration of doping metal is a key factor for successful doping. This optimum value may vary significantly and depends on several factors, such as the type of dopant chosen, the coating deposition technique, annealing conditions, etc. [5].

Pulsed DC reactive magnetron sputtering of a titanium target in an argon/oxygen atmosphere represents a convenient and scalable technique for the deposition of photocatalytic coatings. Doping metals can be easily incorporated into the coating structure by introducing an additional target to the sputtering system. The doping metal content can be varied with good precision by varying the power applied to the doping target. Titania coatings deposited by the magnetron sputtering technique are characterized with high uniformity and good substrate adhesion [6].

Tungsten doping is proven to be an efficient technique for solving the major problems associated with the use of titania as a photocatalyst. Having an ionic radius similar to that of titanium, tungsten is expected to be incorporated into the titania crystal lattice in a substitutional manner [2]. Several authors have reported significant improvements in the photoactivity of titania through doping it with tungsten [3,7-9]. However, information on the optimum tungsten doping level varies significantly in these different works. Thus, Li et al. described titania with 3 at.\% of $\mathrm{W}$ as high activity photocatalyst for coatings deposited by the sol-gel method [10], while Kubacka et al. found the optimum concentration to be significantly higher-19 at.\%, with the coatings prepared by a microemulsion method [7]. It can be seen that the optimum dopant content may vary significantly, depending on the deposition process used. Therefore, this work was conducted to satisfy the need for an optimization study for W-doped coatings deposited by the reactive magnetron co-sputtering technique. The present authors have already highlighted the efficiency of tungsten as a doping metal for titania coatings [2,3], however no systematic study on the doping metal content and its influence on the photocatalytic and structural properties of titania has been carried out to date. 


\section{Experimental Section}

\subsection{Sample Preparation}

The coatings were deposited by magnetron sputtering in a Teer Coatings Ltd. (Droitwich, UK) UDP450 rig from three vertically opposed $300 \mathrm{~mm} \times 100 \mathrm{~mm}$ unbalanced planar magnetrons, which surround a rotating substrate holder. Titanium targets of $99.5 \%$ purity were fitted to two of the magnetrons; the tungsten target (99.99\% purity) was fitted on the third magnetron. The titanium targets were driven in pulsed DC mode using a dual channel Advanced Energy Pinnacle Plus power supply. A time-averaged power of $1 \mathrm{~kW}$ was delivered to each target in synchronous mode at $100 \mathrm{kHz}$ pulse frequency, 50\% duty. The third magnetron, with the tungsten target, was operated in DC mode using an Advanced Energy MDX power supply over the range of powers 60-180 W, to vary the dopant content in the coatings. Undoped titania coatings were also deposited for benchmarking purposes. The reactive sputtering process was carried out in an argon/oxygen atmosphere at a pressure of $0.3 \mathrm{~Pa}$. The oxygen flow rate was controlled using a Reactaflo ${ }^{\mathrm{TM}}$ (Megatech Ltd., Cannock, UK) optical emission monitoring (OEM) system at a set point of $15 \%$ of the full metal, which has been found previously to produce stoichiometric titania coatings [2]. The soda-lime glass substrates used in this work were ultrasonically cleaned in propanol prior to deposition and mounted on the rotatable substrate holder. The rotation speed was set at $4 \mathrm{rpm}$ for all coatings produced in this work.

\subsection{Coating Characterization}

The composition of the as-deposited coatings was analyzed with EDX (EDAX Trident, installed on a Zeiss Supra 40 VP-FEG-SEM, Edax Co., Mahwah, NJ, USA). The coatings were the annealed in air at a temperature of $873 \mathrm{~K}$ for $30 \mathrm{~min}$, followed by gradual cooling (for a total time of 5 hours) to avoid thermally stressing the coatings. All the annealed coatings were analyzed by Raman spectroscopy (RenishawInvia, $514 \mathrm{~nm}$ laser, Renishaw, Gloucestershire, UK) and X-ray diffraction (Philips PW1729 diffractometer with $\mathrm{CuK} \alpha 1$ radiation at $0.514 \mathrm{~nm}$, PANalytical Ltd., Cambridge, UK) for determination of the crystal structure. Coating thickness was measured using Dektak profilometry. The surface roughness of the coatings was determined using MicroXAM white light surface profilometry. TEM analysis was also carried out externally on selected samples (FEI Tecnai FEGTEM Field Emission gun TEM/STEM fitted with HAADF detector, FEI, Cambridge, UK). Prior to TEM analysis, the coatings were thinned with focused ion beam (FIB) and coated with platinum. Platinum layers were used as sacrificial layer to protect the sample of the destructive sputtering of the ions, during FIB.

\subsection{Evaluation of Photocatalytic Activity}

The assessment of the photocatalytic properties of the coatings was carried out using the methylene blue dye photodecomposition technique. Methylene blue (MB), an organic dye with molecular formula $\mathrm{C}_{16} \mathrm{H}_{18} \mathrm{ClN}_{3} \mathrm{~S}$, is often used for photocatalytic tests. ISO standard 10678 confirms the use of methylene blue as a model dye for surface photocatalytic activity determination in aqueous medium [11]. Solutions of MB in distilled water have an intense blue color, and therefore show a strong absorption peak at $665 \mathrm{~nm}$. The concentration of MB solution used in this work was $1.5 \mu \mathrm{mol} / \mathrm{L}$. Changes of the $665 \mathrm{~nm}$ absorption peak 
height were continuously monitored for a total time of 1 hour with an Ocean Optics USB 2000+ spectrometer. According to the Lambert-Beer law, the concentration of dye is proportional to the absorbance value:

$$
A=\varepsilon c l
$$

where $\mathrm{A}$ is absorbance, $\varepsilon$ is the molar absorbance coefficient; $l$ is the optical length of the cell where the photocatalyst is immersed into MB.

The photocatalytic decomposition of MB was approximated to first order kinetics, as shown in the equation:

$$
\ln \left[\frac{C_{0}}{C}\right]=k_{a} t
$$

where $C_{0}$ and $C$ are the concentrations of $\mathrm{MB}$ solution at time 0 and time $t$ of the experiment, respectively.

If the ratio of absorption decay is proportional to the concentration decay, the first order reaction constant, $k_{a}$ can be found from the slope of the plot $\ln \left(A_{0} / A\right)$ against time.

Prior to the photocatalytic experiments, samples of the coatings were immersed in a conditioning solution of $\mathrm{MB}$ for $30 \mathrm{~min}$ in the dark for pre-absorption of the dye on the test surface. For the photocatalytic activity measurements samples were immersed into $40 \mathrm{~mL}$ of $\mathrm{MB}$ aqueous solution of concentration $1.5 \mu \mathrm{mol} / \mathrm{L}$ (the concentration was defined experimentally to be able to detect photocatalytic response of each coating tested in 1 hour experiment). Each coating was tested under UV and fluorescent light sources; $2 \times 15$ W 352 nm Sankyo Denki BLB lamps (Sankyo Denki Co., Osaka, Japan) were used as the UV light source and $2 \times 15$ W Ushio fluorescent lamps (USHIO, Tokyo, Japan) as the fluorescent light source. Figure 1 shows the spectra of the UV and fluorescent tubes used for the photocatalytic tests. The integrated power flux to the coating was $4 \mathrm{~mW} / \mathrm{cm}^{2}$ for the UV light tubes, and $6.4 \mathrm{~mW} / \mathrm{cm}^{2}$ for fluorescent light tubes of which there is a UV component of $0.5 \mathrm{~mW} / \mathrm{cm}^{2}(8 \%)$. The natural decay rate of methylene blue (without the photocatalyst presence) under each type of light source was measured for reference purpose, as well as degradation rate of methylene blue in contact with photocatalytic surface but without light irradiation (in the dark). In both cases the degradation rate of methylene blue during 1-hour experiments was of zero order, thus could be neglected in the following calculations. Therefore any changes in the absorption peak height could only be attributed to the photocatalytic activity of the coating [12].

Figure 1. Spectra of the UV and fluorescent tubes used for photocatalytic testing.

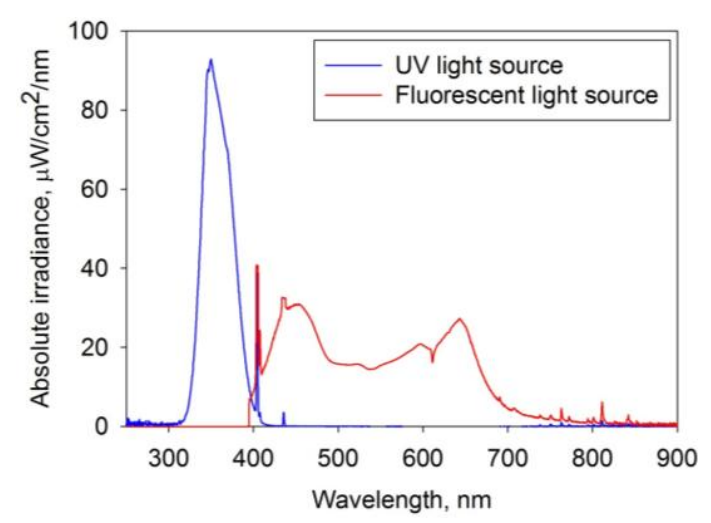




\section{Results and Discussion}

\subsection{Coating Composition and Thickness}

The composition of the coatings, determined by EDX, and their thicknesses are presented in Table 1.

Table 1. Compositional properties and thickness of W-doped titania coatings.

\begin{tabular}{cccc}
\hline Coating ID & Power applied on tungsten target, $\mathbf{W}$ & Tungsten content, at.\% & Coating thickness, nm \\
\hline W60 & 60 & 3.83 & 702 \\
W70 & 70 & 4.64 & 746 \\
W80 & 80 & 5.89 & 758 \\
W90 & 90 & 7.09 & 793 \\
W100 & 100 & 10.03 & 814 \\
W150 & 150 & 13.87 & 889 \\
W180 & 180 & 15.84 & 896 \\
\hline
\end{tabular}

Raman spectroscopy showed that all the as-deposited coatings were amorphous. After annealing in air at a temperature of $873 \mathrm{~K}$ the coatings were re-analyzed by Raman spectroscopy and XRD. Prior to photocatalytic testing, the visual appearance of the coatings was also studied. Coatings W60-W100 were uniform and optically transparent, and essentially identical to undoped titania coatings annealed at the same temperature. Coatings W150 and W180 was bluish in color, with the color being more intense for the coatings containing higher loadings of tungsten. This, along with the thickness of the coatings increasing with increasing dopant content, may indicate the formation of tungsten oxide. This may happen when the concentration of tungsten in the coating exceeds the dispersion limit in titanium dioxide. The bluish color of the coatings can be attributed to the presence of tungsten oxide with molecular formula $\mathrm{W}_{20} \mathrm{O}_{58}$ [13], which can form as a result of the reaction of tungsten ions with amounts of oxygen insufficient for the formation of $\mathrm{WO}_{3}$.

\subsection{Raman Spectroscopy Results}

The results of Raman spectroscopy of the annealed coatings are shown in Figure 2a,b. As can be seen from the Raman spectra, all the annealed coatings were crystalline. However significant differences were observed in the spectra for coatings with lower (W60-W90) and higher (W100-W180) tungsten content. The lower tungsten content coatings showed intense peaks at 144, 397, 516 and $638 \mathrm{~cm}^{-1}$ which can be directly attributed to the anatase phase. All the coatings also had a weak, broad peak at $968 \mathrm{~cm}^{-1}$ which can be attributed to $\mathrm{W}=\mathrm{O}$ bond stretching [14], which is common for all types of tungsten oxide. Increasing the tungsten content resulted in weaker crystal structures with broader peaks. The crystal structure of coatings W100 can be described as mixed anatase/rutile, with anatase peaks as described earlier and rutile peaks at 448 and $609 \mathrm{~cm}^{-1}$. Samples W150 and W180 exhibited only rutile peaks. The broad peak at $273 \mathrm{~cm}^{-1}$ can be attributed to the presence of tungsten oxide species on the surface of the coatings [13]. This peak is absent from the spectra of coatings W60-W90, however, it may be hidden by the high intensity of the anatase peaks. 
Figure 2. Results of Raman spectroscopy of titania and W-doped titania coatings deposited onto glass substrates and annealed at $873 \mathrm{~K}$ (a) $\mathrm{TiO}_{2}$ and samples W60-W90; (b) Samples W100-W180.

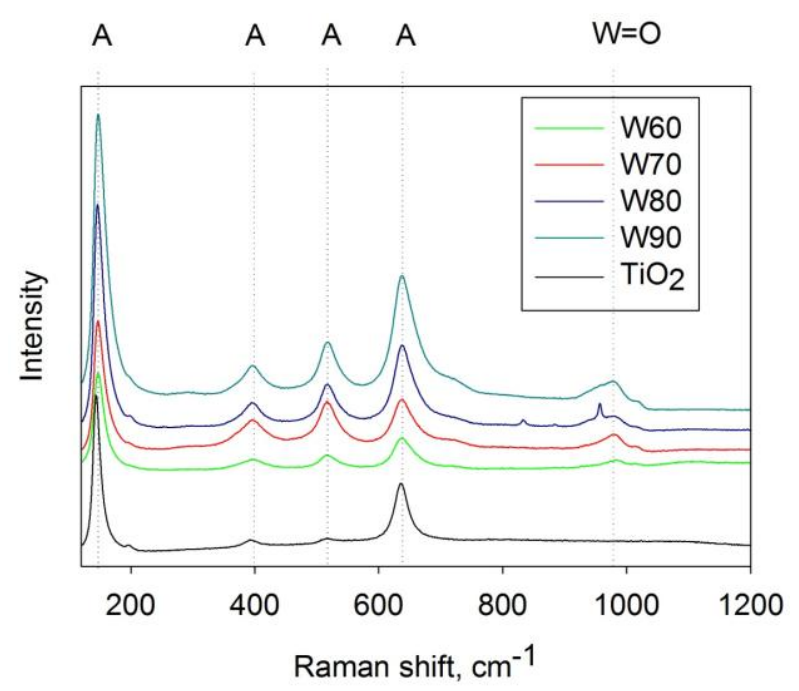

(a)

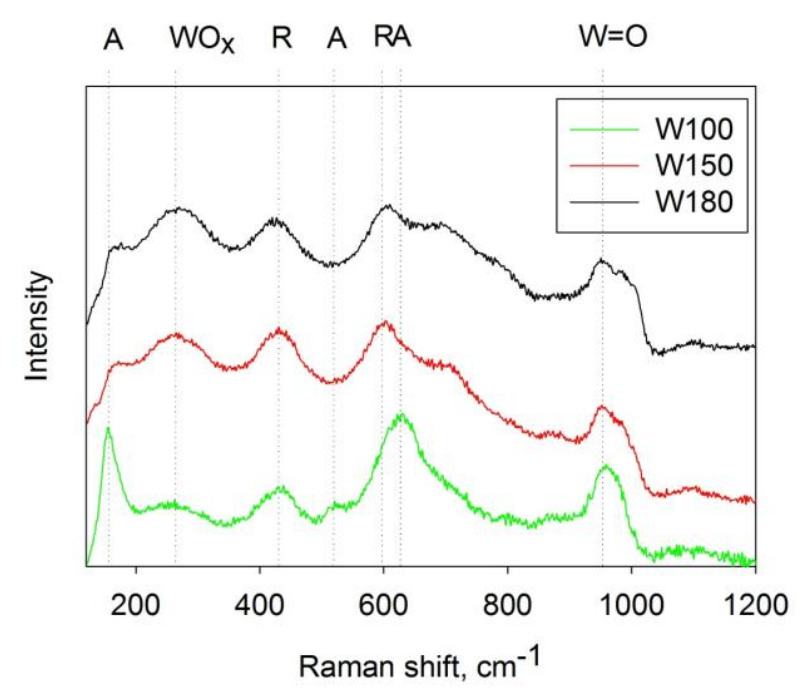

(b)

The Raman spectrum for an undoped titania coating annealed at a corresponding temperature is presented for comparison purposes. It can be seen that pure titania also has an anatase structure, however, unlike the W-doped coatings, no other peaks can be seen in the spectrum.

Overall, the Raman spectra of W-doped coatings clearly indicate that increasing W content leads to the formation of a rutile structure, with sample W180 showing rutile peaks only. Some proportion of the added tungsten forms an oxide on the surface of the coatings; this can be seen more clearly for the coatings with higher $\mathrm{W}$ content.

\subsection{XRD Results}

The XRD patterns obtained for the annealed W-doped titania coatings are presented in Figure 3a,b. As for the results of Raman spectroscopy, they are divided into two parts for ease of comparison. An XRD pattern for undoped titania is also presented for reference purposes. It can be seen that both 
undoped and all of the W-doped coatings were crystalline after annealing at $873 \mathrm{~K}$. Coatings W60-W100 were in the anatase phase with major anatase peaks (marked $\mathrm{A}$ in the figure) found at $25.3^{\circ}$, $37.8^{\circ}, 48.0^{\circ}, 55.0^{\circ}$ and $62.6^{\circ} 2 \theta$ (JCPDS: 21-1272). Coatings W150 and W180 showed the presence of characteristic rutile peaks (marked R) at $27.4^{\circ}, 36.0^{\circ}, 39.1^{\circ}, 54.3^{\circ}, 56.6^{\circ}$ and $64.0^{\circ} 2 \theta$ (JCPDS: 21-1276) with W150 being a mix of anatase and rutile and W180-purely rutile. Generally, XRD data were in good agreement with Raman spectroscopy data. However, no peaks besides those corresponding to titania were found in any of the XRD patterns obtained. It is presumed that the absence of any tungsten oxide peaks is due to the amount present being below the detection limit of the XRD system available.

Figure 3. XRD results of titania and W-doped titania coatings deposited onto glass substrates and annealed at $873 \mathrm{~K}$. (a) $\mathrm{TiO}_{2}$ and samples W60-W90; (b) Samples W100-W180.

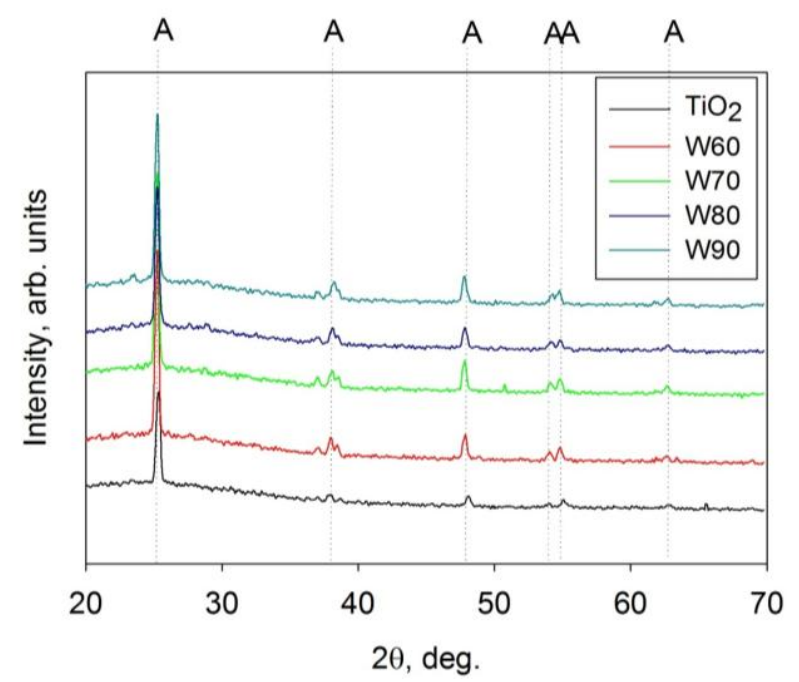

(a)

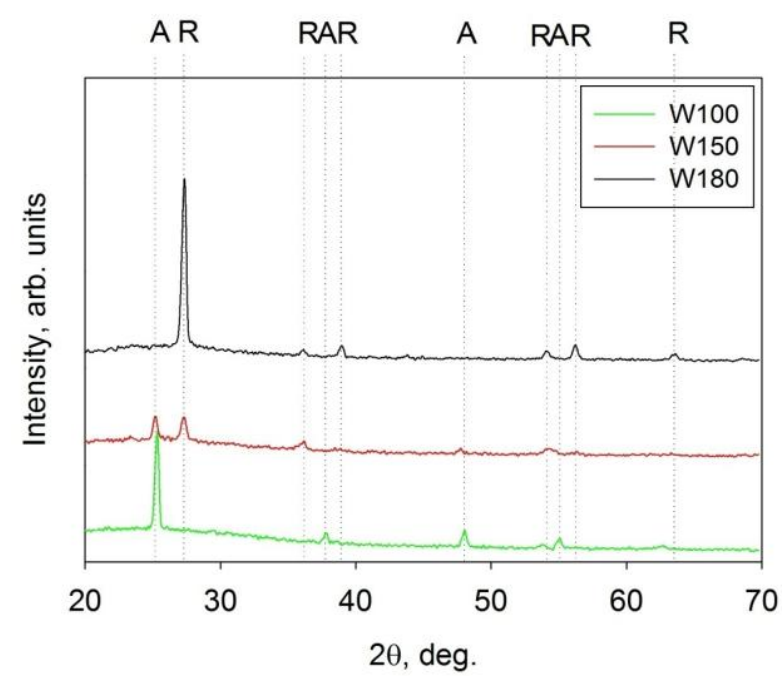

(b)

\subsection{Band Gap Calculation}

Values of the optical band gaps of the coatings were calculated using the Tauc plot method [15], by plotting $(\alpha h v)^{1 / 2}$ versus $h v$ and extrapolating the linear region to the abscissa (where $\alpha$ is absorbance 
coefficient, $h$-Plank's constant, $v$ is the frequency of vibration). Examples of this method are shown graphically in Figure 4, and band gap values for all coatings studied are presented in Table 2, along with other analytical results.

As can be seen from the results, increasing tungsten content results in significant band gap shifts towards the visible range. However, narrowing of the band gap may also be partly attributed to crystal phase transitions of titanium dioxide. It is known that the band gap value of pure anatase titania is $3.2 \mathrm{eV}$, while for the pure rutile phase this value is somewhat lower $(3.1 \mathrm{eV})$. As shown from the results of XRD and Raman spectroscopy, coatings with higher W content tended to form the rutile crystal phase. Thus, some proportion of the decrease of the band gap value for the high $\mathrm{W}$-content coatings must be attributed to these phase transformations. For coatings W60-W90 with lower W-content and anatase structures, the red shift of the band gap caused by the presence of tungsten can be evaluated as being moderate, with the absorbance limit shifted from $392 \mathrm{~nm}$ for undoped titania to $406 \mathrm{~nm}$ for sample W90.

Figure 4. An example of optical band gap calculation for undoped and W-doped titania coatings annealed at $873 \mathrm{~K}$.

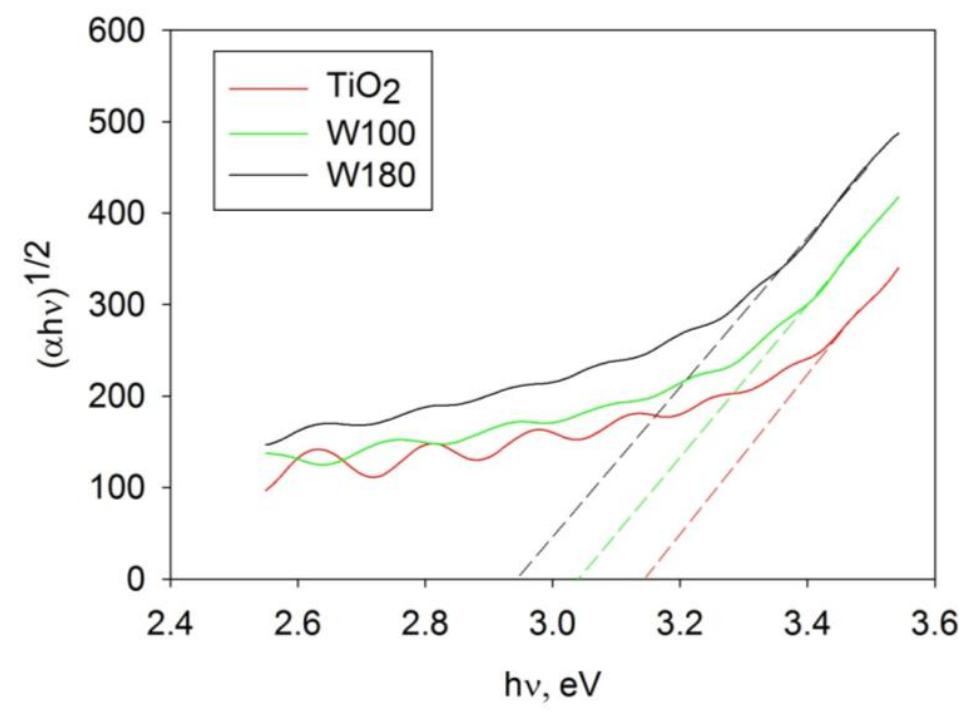

Table 2. Morphological characteristics, photocatalytic activity values and optical band gaps of titania and W-doped titania coatings after annealing at $873 \mathrm{~K}$.

\begin{tabular}{ccccccc}
\hline Coating ID & $\begin{array}{c}\text { Band gap, } \\
\mathbf{e V}\end{array}$ & $\begin{array}{c}\boldsymbol{k}_{\boldsymbol{a}} \times \mathbf{1 0}^{\mathbf{5}} \mathbf{\mathbf { s } ^ { - \mathbf { 1 } }} \\
\mathbf{U V} \text { light }\end{array}$ & $\begin{array}{c}\boldsymbol{k}_{\boldsymbol{a}} \times \mathbf{1 0}^{\mathbf{5}}, \mathbf{s}^{\mathbf{- 1}} \\
\text { Fluorescent light }\end{array}$ & $\begin{array}{c}\text { Surface } \\
\text { roughness, } \boldsymbol{\mu m}\end{array}$ & $\begin{array}{c}\text { Surface } \\
\text { area, } \boldsymbol{\mu m}^{2}\end{array}$ & $\begin{array}{c}\text { Crystal } \\
\text { structure }\end{array}$ \\
\hline $\mathrm{TiO}_{2}$ & 3.16 & 1.7 & 0.6 & 0.0114 & 5523 & anatase \\
$\mathrm{W} 60$ & 3.12 & 4.0 & 1.0 & 0.0491 & 5620 & anatase \\
$\mathrm{W} 70$ & 3.09 & 5.6 & 1.2 & 0.0309 & 5614 & anatase \\
$\mathrm{W} 80$ & 3.09 & 9.9 & 2.7 & 0.0218 & 5602 & anatase \\
$\mathrm{W} 90$ & 3.05 & 6.4 & 2.1 & 0.0161 & 5554 & anatase \\
$\mathrm{W} 100$ & 3.02 & 2.2 & 1.6 & 0.0144 & 5535 & anatase \\
$\mathrm{W} 150$ & 3.00 & 1.4 & 0.8 & 0.0138 & 5525 & anatase/rutile \\
$\mathrm{W} 180$ & 2.98 & 0.9 & 0.6 & 0.0152 & 5520 & rutile \\
\hline
\end{tabular}




\subsection{Morphological Properties}

The morphological properties of the coatings (surface roughness and surface area), evaluated by white light surface profilometry can be seen in Table 2. There is a clear trend in the results of these measurements, with sharp increases in surface roughness and surface area with $\mathrm{W}$ content for coatings W60 to, compared to undoped titania. However, with further increases of tungsten the surface area of the coatings was found to reduce back to the values obtained for undoped titanium dioxide. Examples of 3D surface images can be seen in Figure 5a-c. High values of surface roughness and surface area are often described to be the distinctive features of $\mathrm{W}$-doped titania coatings, regardless the deposition method used $[16,17]$. High coating surface area is believed to contribute to the photocatalytic activity observed, as it increases the area in contact with the methylene blue. The decrease of surface area and surface roughness values for the high $\mathrm{W}$-content coatings may be a result covering of the surface of the photocatalyst with tungsten oxide species, as indicated by the Raman results.

Figure 5. 3D surface maps (autoscaled along $Z$ axis) obtained by white light surface profilometry. (a) undoped titania; (b) sample W60; (c) sample W100.

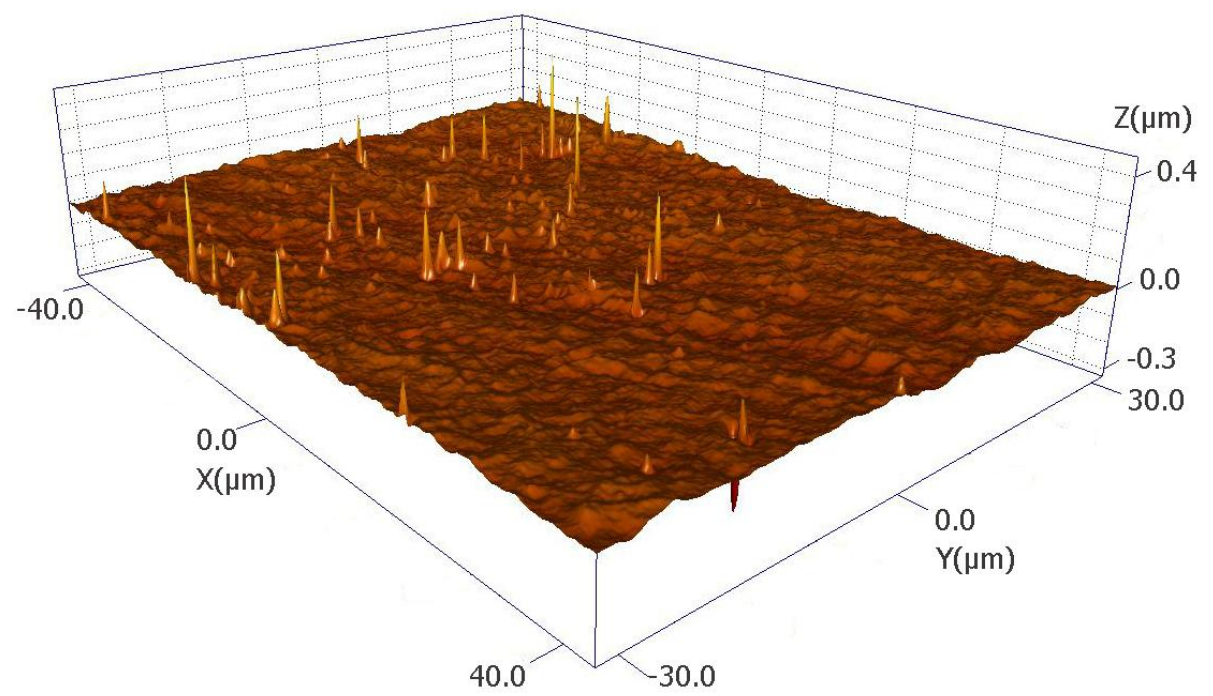

(a)

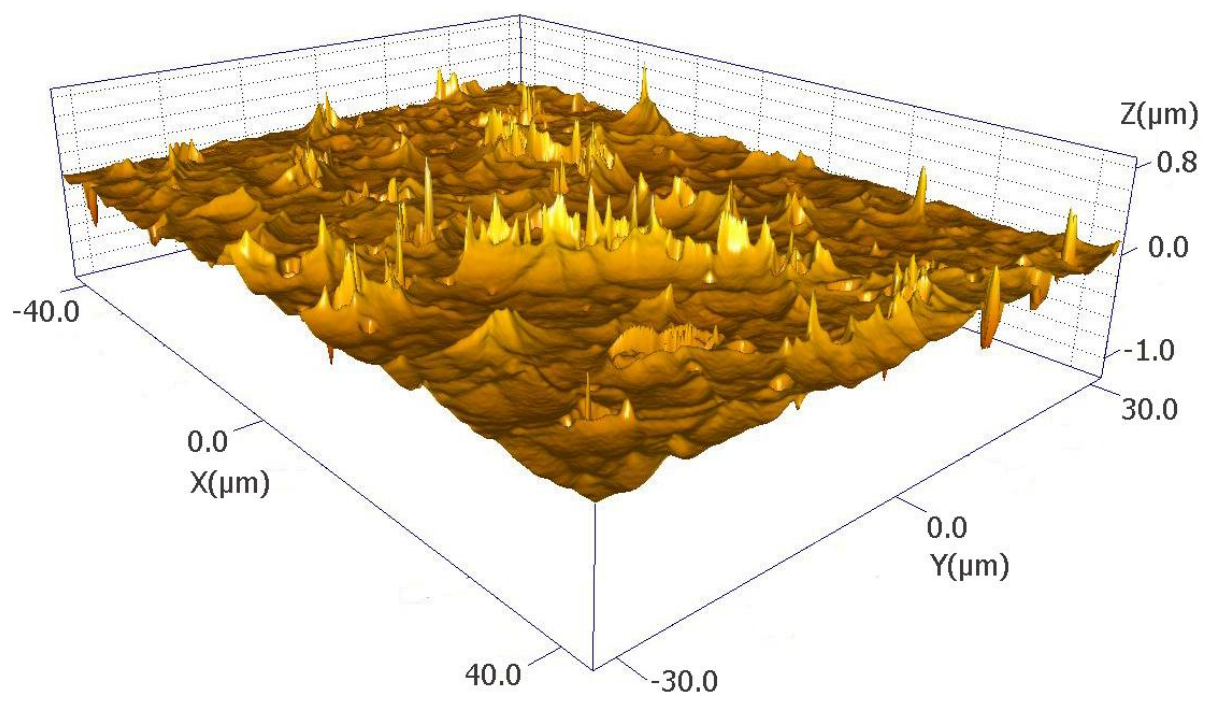

(b) 
Figure 5. Cont.

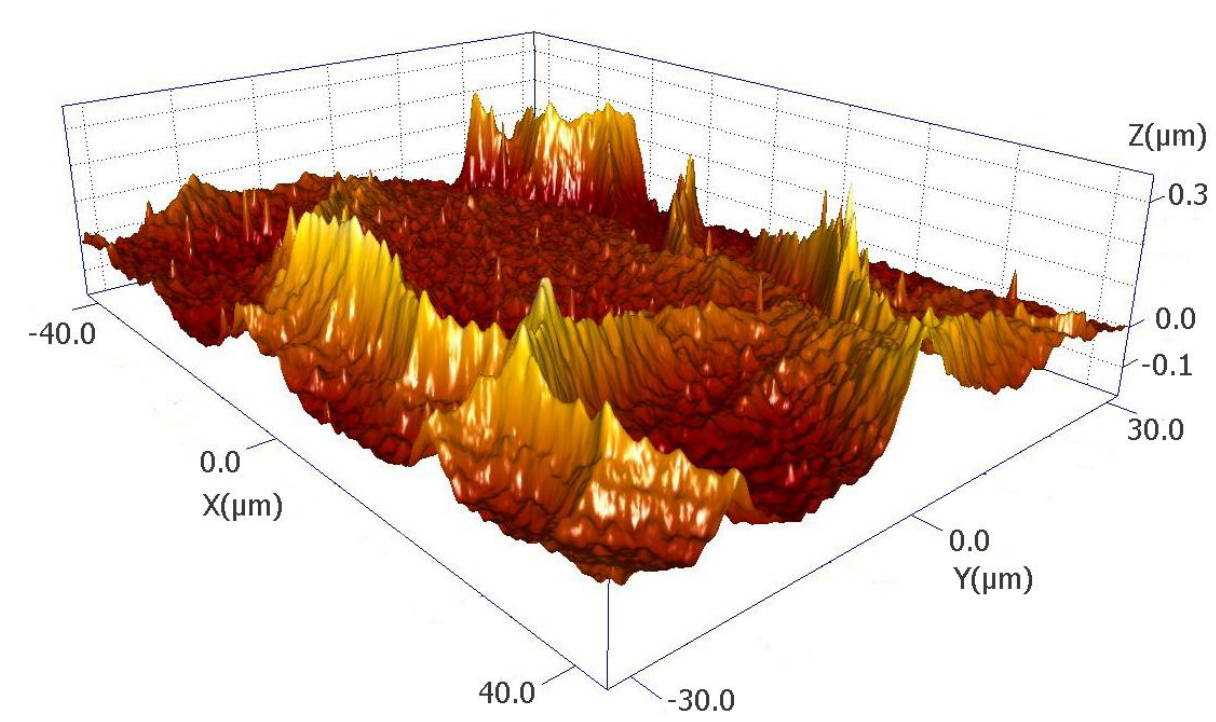

(c)

\subsection{TEM Results}

TEM analysis of selected coatings was carried out in order to investigate the structural properties of the coatings and the influence of tungsten doping on the coatings structure. Prior to TEM analysis, the coatings were thinned with a focused ion beam (FIB) and coated with platinum. The platinum layer was used as a sacrificial layer to protect the sample from the destructive sputtering during FIB preparation.

The bright field TEM micrographs obtained are presented in Figure 6a-d. The dependence of the structural changes observed on the tungsten content can be seen in these micrographs. Figure 6a shows a TEM micrograph of an undoped titania coating. The structure presents a relatively dense mixture of fine and coarse crystals. The layer seen on the bottom of the coating consists of amorphous titania, as indicated from diffraction results (shown in Figure 6a). The micrograph of sample W80 is shown in the Figure $6 \mathrm{~b}$, revealing a fine columnar structure for this coating. It can be seen that the base layer of the coating is significantly more porous than the rest of the coating. The diffraction pattern of sample W80 showed that the base layer was microcrystalline rather than amorphous. The TEM micrograph of sample W100 (Figure 6c) showed a nanocrystalline structure. Finally, Coating W150 (Figure 6d) had a uniform fine columnar structure. No base layer or additional crystal inclusions were observed in this sample.

EDX elemental mapping of the doped samples showed a uniform distribution of tungsten throughout the bulk of the coatings.

\subsection{Photocatalytic Activity Measurements}

The values of the first order rate constants for MB photodecomposition under UV and fluorescent light sources are shown in Table 2. It can be seen that considerable improvement in photocatalytic activity was observed with increasing tungsten content, reaching a maximum for sample W80, beyond which the activity levels fell back to the undoped level, or below. Variation in the MB decomposition rate as a function of $\mathrm{W}$ content in the coatings is presented in Figure 7. The curve has a sharp maximum at 5.89 at.\% of $\mathrm{W}$ for either light source. 
Figure 6. TEM micrographs obtained for undoped and W-doped titania coatings annealed at 873 K. (a) Undoped $\mathrm{TiO}_{2}$; (b) Sample W80; (c) Sample W100; (d) Sample W150.
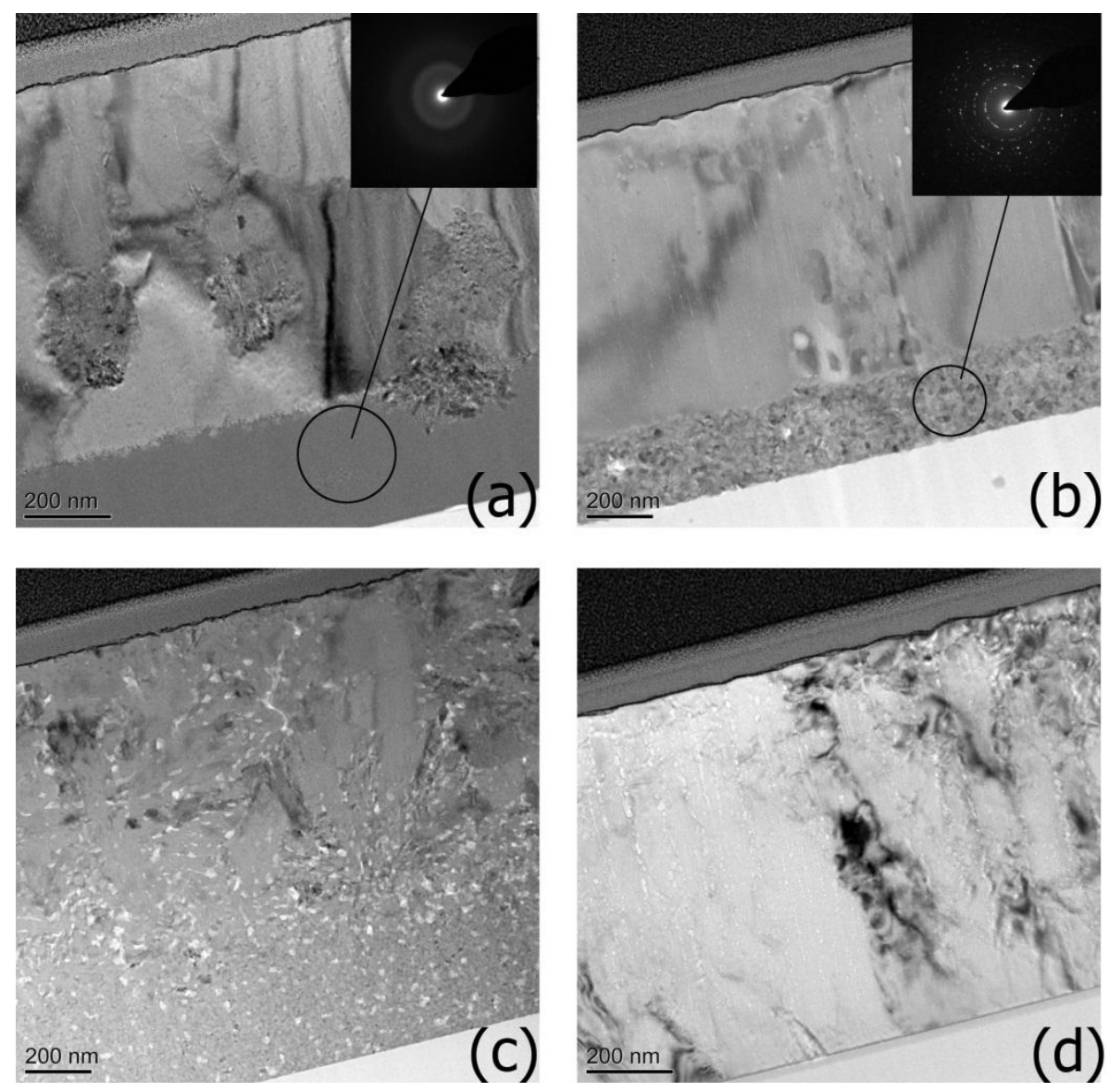

Figure 7. Efficiency of MB degradation by W-doped titania coatings annealed at $873 \mathrm{~K}$ as a function of $\mathrm{W}$ content.

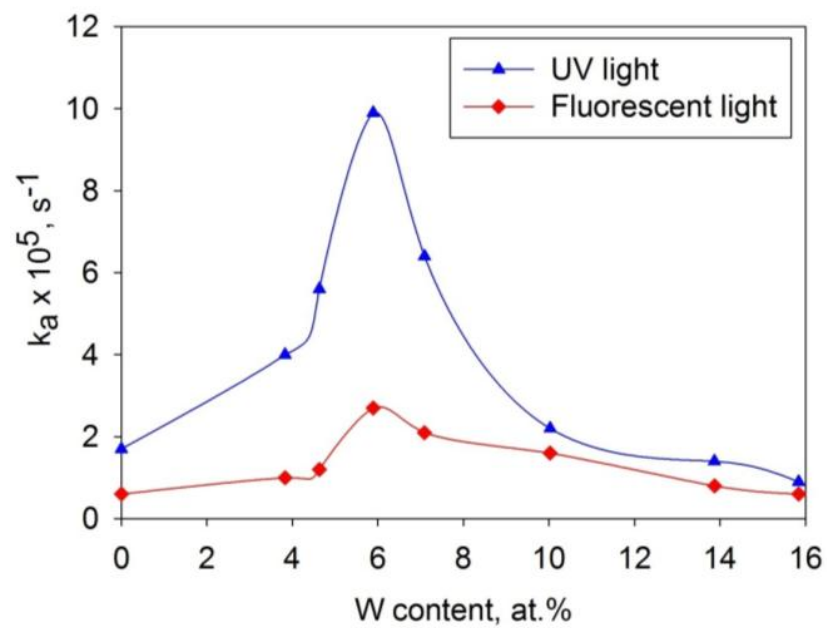

As the band gap shift of the coatings showing the highest activity was relatively small, the increased photocatalytic activity both under UV and fluorescent light sources is believed to be predominantly attributed to improved electron-hole separation, rather than the band gap shift towards the visible range. This can be confirmed by using a UV filter (filter cuts off the light with wavelength less than $400 \mathrm{~nm}$ ) - the use of the filter in photocatalytic measurements significantly 
reduced the level of activity observed for these coatings under the fluorescent light source. Thus, MB decomposition rate constant under the visible light source for the coating W80 was only $0.4 \times 10^{-5} \mathrm{~s}^{-1}$, while for the rest of the coatings these values were even lower.

Since some tungsten oxide was found to exist as a separate phase (according to the Raman spectroscopy results), the following explanation of improved photocatalytic properties of the coatings is proposed. The band gap value of tungsten oxide is reported to be significantly lower than that of titanium dioxide $(2.5-2.8 \mathrm{eV}$ depending on the source $[18,19])$, therefore the conduction and the valence bands of $\mathrm{WO}_{\mathrm{x}}$ are located lower than the corresponding bands of titania. The model presented in Figure 8 is proposed by several authors to represent the photocatalytic mechanism of the W-doped $\mathrm{TiO}_{2}$ photocatalysts [5]. It is also suitable for the explanation of improved photocatalytic behavior observed during this work for coatings deposited by magnetron sputtering. When the photocatalyst is irradiated with light, the photogenerated electrons will be transferred into the tungsten oxide conduction band, as it is located lower than the corresponding band of titanium dioxide. Conversely, the holes will be accumulated on the valence band of titania, assisting the efficient charge separation. In the case of coatings with higher $\mathrm{W}$ content, excessive levels of dopant act as recombination centres for photogenerated elections and holes. In addition, the formation of a separate phase of tungsten oxide reduces the surface area of titanium dioxide, as proved by the surface morphology results, and thus reduces the area of contact between the pollutant and the photocatalyst. These factors result in a significant loss of photocatalytic activity for coatings W150-W180. As the results obtained for both UV and fluorescent light sources showed that the maximum activity occurred at the same tungsten concentration, this can be taken as a proof of more efficient charge carrier separation at 5.89 at.\% of W.

Figure 8. Electron-hole transfer scheme for W-doped titania photocatalysts.

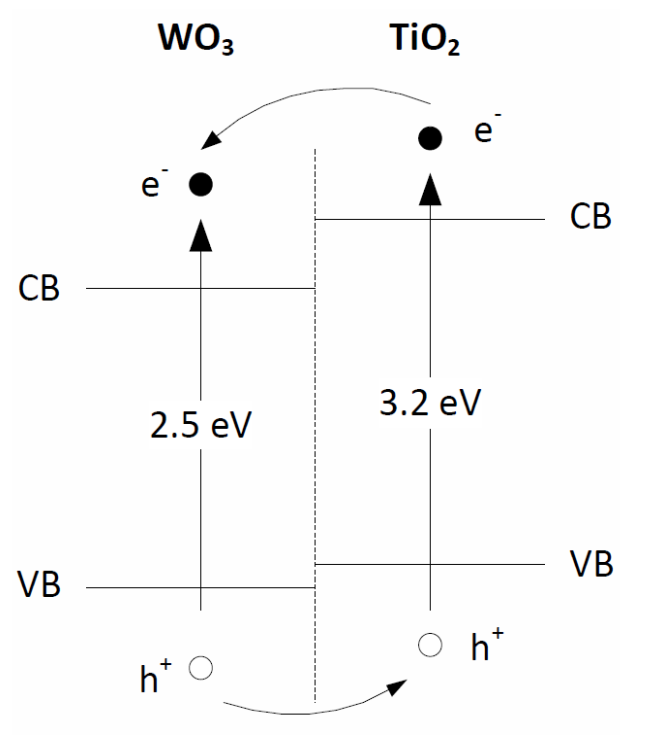

\section{Conclusions}

$\mathrm{W}$-doped titanium dioxide coatings were deposited onto glass substrates by reactive co-magnetron sputtering and their ability to decompose MB under UV/fluorescent light irradiation was successfully demonstrated. For both light sources, low concentrations of tungsten caused significant improvements of 
the photocatalytic properties. The maximum photocatalytic efficiency was found for the coatings containing 5.89 at.\% of tungsten, which can, therefore, be defined as the optimum doping level. The activity of the coating with this optimum level of dopant exceeded that of undoped titania by a factor of 6 under UV light and by a factor of 4 under fluorescent light. The high photocatalytic activity of $\mathrm{W}$-doped titania ( $<10$ at.\% W) coatings is believed to be a result of more efficient electron-hole separation, compared to undoped titania, and higher surface areas for the reaction to take place. Further increase in tungsten content resulted in rutile phase formation and fast charge carrier recombination at the excessive dopant centres, hence very low photocatalytic efficiency.

The shift of the optical band gap of the W-doped titania coatings was found to be quite low, therefore high activity under the fluorescent light source can rather be attributed to the UV part of the spectra, than improved visible light performance. However, under normal indoor irradiation the coatings demonstrated significant photocatalytic efficiency, comparable to the photocatalytic efficiency of undoped titania under UV light. Therefore, despite the low shift in band gap, W-doped coatings with the optimum level of dopant may find their application as efficient indoor photocatalytic materials.

\section{Acknowledgments}

Acknowledgement to Leeds EPSRC Nanoscience and Nanotechnology Research Equipment Facility (LENNF) for conducting the TEM analysis of the coatings.

\section{Conflicts of Interest}

The authors declare no conflict of interest.

\section{References}

1. Rauf, M.A.; Meetani, M.A.; Hisaindee, S. An overview on the photocatalytic degradation of azo dyes in the presence of $\mathrm{TiO}_{2}$ doped with selective transition metals. Desalination 2011, 276, 13-27.

2. Ratova, M.; Kelly, P.J.; West, G.T.; Iordanova, I. Enhanced properties of magnetron sputtered photocatalytic coatings via transition metal doping. Surf. Coat. Technol. 2013, 228, S544-S549.

3. Farahani, N.; Kelly, P.J.; West, G.; Ratova, M.; Hill, C.; Vishnyakov, V. Photocatalytic activity of reactively sputtered and directly sputtered titania coatings. Thin Solid Films 2011, 520, 1464-1469.

4. Yao, M.; Chen, J.; Zhao, C.; Chen, Y. Photocatalytic activities of ion doped $\mathrm{TiO}_{2}$ thin films when prepared on different substrates. Thin Solid Films 2009, 517, 5994-5999.

5. Lorret, O.; Francová, D.; Waldner, G.; Stelzer, N. W-doped titania nanoparticles for UV and visible-light photocatalytic reactions. Appl. Cat. B Environ. 2009, 91, 39-46.

6. Kelly, P.J.; Beevers, C.F.; Henderson, P.S.; Arnell, R.D.; Bradley, J.W.; Bäcker, H. A comparison of the properties of titanium-based films produced by pulsed and continuous DC magnetron sputtering. Surf. Coat. Technol. 2003, 174-175, 795-800.

7. Kubacka, A.; Colón, G.; Fernández-García, M. Cationic (V, Mo, Nb, W) doping of $\mathrm{TiO}_{2}$-anatase: A real alternative for visible light-driven photocatalysts. Cat. Today 2009, 143, 286-292.

8. Abdullah, M.A.; Chong, F.K. Dual-effects of adsorption and photodegradation of methylene blue by tungsten-loaded titanium dioxide. Chem. Eng. J. 2010, 158, 418-425. 
9. Rampaul, A.; Parkin, I.P.; O’Neill, S.A.; DeSouza, J.; Mills, A.; Elliott, N. Titania and tungsten doped titania thin films on glass; active photocatalysts. Polyhedron 2003, 22, 35-44.

10. Li, X.Z.; Li, F.B.; Yang, C.L.; Ge, W.K. Photocatalytic activity of $\mathrm{WO}_{x}-\mathrm{TiO}_{2}$ under visible light irradiation. J. Photochem. Photobiol. A Chem. 2001, 141, 209-217.

11. ISO10678, Fine Ceramics, Advanced Technical Ceramics-Determination of Photocatalytic Activity of Surfaces in an Aqueous Medium by Degradation of Methylene Blue; ISO: Geneva, Switzerland, 2010.

12. Stengl, V.; Bakardjieva, S.; Murafa, N. Preparation and photocatalytic activity of rare earth doped $\mathrm{TiO}_{2}$ nanoparticles. Mat. Chem. Phys. 2009, 114, 217-226.

13. Abdullah, M.A.; Chong, F.K. Preparation and characterization of tungsten-loaded titanium dioxide photocatalyst for enhanced dye degradation. J. Hazard. Mat. 2010, 176, 451-458.

14. Fuerte, A.; Hernandez-Alonso, M.D.; Maira, A.J.; Martinez-Arias, A.; Fernandez-Garcia, M.; Conesa, J.C.; Soria, J. Visible light-activated nanosized doped- $\mathrm{TiO}_{2}$ photocatalysts. Chem. Commun. 2001, 2718-2719.

15. Tauc, J.; Grigorovici, R.; Vancu, A. Optical properties and electronic structure of amorphous germanium. Phys. Stat. Solid. B 1966, 15, 627-637.

16. Kiriakidou, F.; Kondarides, D.I.; Verykios, X.E. The effect of operational parameters and $\mathrm{TiO}_{2}$-doping on the photocatalytic degradation of azo-dyes. Cat. Today 1999, 54, 119-130.

17. Imai, M.; Kikuchi, M.; Oka, N.; Shigesato, Y. Visible light-induced photocatalytic properties of $\mathrm{WO}_{3}$ films deposited by DC reactive magnetron sputtering. J. Vac. Sci. Technol. A Vac. Surf. Films 2012, 30, 031503.

18. Hathway, T.; Rockafellow, E.M.; Oh, Y.-C.; Jenks, W.S. Photocatalytic degradation using tungsten-modified $\mathrm{TiO}_{2}$ and visible light: Kinetic and mechanistic effects using multiple catalyst doping strategies. J. Photochem. Photobiol. A Chem. 2009, 207, 197-203.

19. Biswas, S.; Hossain, M.F.; Shahjahan, M.; Takahashi, K.; Takahashi, T.; Fujishima, A. Investigation of photocatalytic activity of $\mathrm{TiO}_{2} / \mathrm{WO}_{3}$ bilayered thin films with various amounts of $\mathrm{WO}_{3}$ exposed surface. Am. Vac. Soc. 2009, 27, 880-884.

(C) 2013 by the authors; licensee MDPI, Basel, Switzerland. This article is an open access article distributed under the terms and conditions of the Creative Commons Attribution license (http://creativecommons.org/licenses/by/3.0/). 